\title{
LASSI: METRIC BASED I/O ANALYTICS FOR HPC
}

\author{
Karthee Sivalingam \\ Harvey Richardson \\ Adrian Tate \\ Cray European Research Lab \\ Broad Quay House, Prince Street \\ Bristol, UK \\ \{ksivalinga,harveyr, adrian\}@ cray.com
}

Martin Lafferty

Cray, UK

ACF Building

Penicuik, UK

rml@cray.com

\begin{abstract}
LASSi is a tool aimed at analyzing application usage and contention caused by use of shared resources (filesystem or network) in a HPC system. LASSi was initially developed to support the ARCHER system where there are large variations in application requirements and occasional user complaints regarding filesystem performance manifested by variation in job runtimes or poor interactive response. LASSi takes an approach of defining derivative risk and ops metrics that relate to unusually high application I/O behaviour. The metrics are shown to correlate to applications that can experience variable performance or that may impact the performance of other applications. LASSi uses I/O statistics over time to provide application I/O profiles and has been automated to generate daily reports for ARCHER. We demonstrate how LASSi provides holistic I/O analysis by monitoring filesystem I/O, generating coarse profiles of filesystems and application runs and automating analysis of application slowdown using metrics.
\end{abstract}

Keywords: I/O, ARCHER, Slowdown, Lustre, Monitoring, Metrics

\section{INTRODUCTION}

High Performance Computing (HPC) jobs are usually scheduled to run on dedicated compute nodes, but will share certain hardware resources with other jobs. In particular, the high-performance interconnect and I/O systems of a supercomputer are typically shared, and so contention can occur when multiple applications/users access these shared resources simultaneously. Shared resources can also be used inefficiently, for example pathologically bad patterns of communication (affecting the network) or inefficient I/O (high metadata rate requirements or small-sized I/O operations) (National Computational Infrastructure 2018, NICS 2018, NASA GOV 2018). The combination of these two situations is that poor usage on the part of one user can negatively affect the performance of the shared resource for other users. Users expect consistent runtimes but sizing and operating a system to deliver this on an unknown and varied workload is very difficult, especially regarding shared resources. In extreme cases user jobs can fail by running unexpectedly past the wallclock time limit requested by the user, resulting in loss of simulation data. Users are reluctant to deal with this by, for example, checkpointing.

LASSi provides HPC system support staff the ability to $a$ ) monitor and profile the I/O usage of applications over time $b$ ) identify and study metrics displaying the quantity and quality of application I/O over time c) study the risk of slowdown for applications at any time and identify causes for high risk $d$ ) study rogue 
applications in detail using profiling tools to identify issues at the application level and suggest functional or code changes. LASSi aims to provide early warning and health status metrics to support staff, enabling much faster triaging of potential I/O issues and the high-level diagnosis of I/O problems.

\subsection{Background}

The UK's national supercomputing service ARCHER (https://www.archer.ac.uk) supports a highly-varied workload of applications from a range of disciplines including Weather \& Climate, Materials Science, Computational Chemistry, Computational Fluid Dynamics, Turbulence research, Quantum Mechanics, High Energy Physics, Biomolecular simulation and Mesoscale engineering along with emerging technologies in AI and Data science. These applications have different compute and data requirements but share a common Lustre (Braam et al. 2003) file system. This sharing can introduce contention that may impact performance. The severity of the performance impact can be severe enough to affect a user's ability to list directory information.

Users can be quite sensitive to runtime variation or slowdown of submitted jobs. Application owners usually submit many similar jobs and expect them to complete on time. A slowdown event is when a few loosely concurrent jobs run slower than their respective expected runtimes. Unfortunately, there is no more precise definition of expected runtime than to roughly correspond to the user's wishes. ARCHER support staff have the responsibility to analyse the reasons for slowdown and then suggest corrective actions. Slowdown can be attributed to many factors that also include changes in scientific configuration, node configuration, filesystem load and network traffic. It has been observed that a few rogue applications may cause slowdown for all users. The diverse workload running on ARCHER does not allow a single solution for all such issues.

ARCHER supports many application that are I/O bound and a detailed study the system's I/O load (Turner et al. 2017) has discussed which file layouts and Lustre striping settings are to be used for optimal performance and scaling. Many efforts have been made to educate the community through lectures and training events (Henty 2018, EPCC 2018). Although these activities are helpful, problems continue to be seen and it is important to focus on problem remediation as well as I/O optimization.

Analysing the slowdown of applications and modeling runtime of jobs in a HPC system is highly complex and time-consuming. Thus, slowdown events incur a high cost to any HPC site or service provider in terms of staff time. LASSi was developed to vastly decrease the amount of time and effort (and cost) required to detect, diagnose and remediate such issues.

\section{I/O MONITORING AND STATISTICS}

LASSi combines Lustre statistics and job information in order to calculate derived metrics. I/O statistics are collected using a bespoke tool called LAPCAT which in turn uses Cerebro (https://github.com/lmenezes/cerebro) to collect Lustre statistics, storing them in a MySql database on a management server. LAPCAT was developed by Martin Lafferty of Cray UK. Job information is obtained from the job scheduler and ALPS (Karo, Lagerstrom, Kohnke, and Albing 2006) logs. On ARCHER, LASSi combines the per-node I/O statistics with the job time information to attribute I/O statistics to individual application launches. The jobstats feature available in newer versions of Lustre can provide some of this information. 
Sivalingam, Richardson, Tate and Lafferty

\subsection{ARCHER}

ARCHER is the UK's national supercomputing facility and is a Cray XC30 (Cray 2018) supercomputer. A high-performance Lustre storage system is available to all compute nodes and is based on Cray Sonexion 1600 storage running Lustre 2.1. This storage system provides 4 filesystems configured from multiple storage units - Object Storage Targets (OSTs). The $f_{s} 1$ filesystem has 8 OSTs, $f_{s} 2$ has 48 OSTs, $f_{s} 3$ has 48 OSTs and $f s 4$ has 56 OSTs. These filesystems have to support the wide variety of application domains which produce a complex workload with varying I/O requirements at any given time.

Application runtimes are a function of many factors that include compute clock speed, memory bandwidth, I/O bandwidth, network bandwidth and scientific configuration (dataset size or complexity). Application run time variations due to change in compute resource and memory can be ignored. The I/O system and network are shared resources and are the main causes of slowdown whereas changes to scientific configuration are beyond the scope of LASSi.

\subsection{Lustre}

Lustre is a distributed parallel filesystem with two important components: the Object Storage Server (OSS) and the MetaData Server (MDS). The I/O operation statistics on each server can be used to study application I/O usage/performance. LASSi uses the following I/O statistics: a) OSS: read_kb, read_ops, write_kb, write_ops, other b) MDS: open, close, mknod, link, unlink, mkdir, rmdir, ren, getattr, setattr, getxattr, setxattr, statfs, sync, sdr, cdr.

Statistics are aggregated over a time window of three minutes by LAPCAT. The OSS provides bulk data storage for applications to store data in files. Statistics read_kb and write_kb refer to the amount of data read and written respectively, while read_ops and write_ops refer to the number of Lustre operations that are used to achieve corresponding read and writes. The statistic other in OSS refers to the sum of get_info, set_info_async, disconnect, destroy, punch, sync, preprw and commitrw operations - all relating to the reading and writing of data on the OSS. The MDS operations relate to filesystem metadata information like file open and close. The MDS supports creating and deleting objects and controlling application's access to files. Lustre servers provide statistics for both OSS and MDS operations in stats files on the filesystem.

\subsection{I/O Statistics}

ARCHER I/O statistics covering a period of 15 months were collected. Initial analysis of the raw statistics revealed great complexity of filesystem usage and individual application I/O profiles. LASSi derives higherlevel and more practically useful metrics than the raw I/O statistics. At a basic level, the Relative Standard Deviation (RSD), a common measure of dispersion of a probability distribution, is calculated for each $\mathrm{I} / \mathrm{O}$ statistic as follows:

$$
c_{v}=\frac{\sigma}{\mu}
$$

where $\sigma$ and $\mu$ are the standard deviation and mean of the data, respectively. Some I/O statistics such as getxattr, setxattr, $s d r$ and $c d r$ are ignored as previous experience shows that they are not prominent. Tables 1 and 2 show the Lustre statistics of the OSS and MDS respectively for a particular I/O operation that are accumulated per hour. For example on $f s 2$, applications create 105 directories per hour with RSD of 130 . A distribution is considered to be low variance if RSD is less than 1 and so a large RSD value signales a high variance an I/O statistic. On ARCHER we generally see a high variance in I/O statistics. For OSS operational statistics, $f_{s} 3$ shows very high variance compared to $f_{s} 4$ and $f_{s} 2$. For MDS operational statistics, $f_{s} 2$ shows higher variance than $f_{s} 3$ and $f_{s} 4$. 
$f_{S} 1$ is used for training and we will ignore herein. In terms of application hours, $f_{S} 3$ is used roughly twice as heavily as other filesystems. The OSS statistics show a mixed picture, with more reads onto $f s 4$ and more writes onto $f_{s} 3$. Looking at the sum of all MDS operations, $f_{s} 4$ sees almost twice as many as $f_{s} 3$ or $f_{s} 2$.

Table 1: OSS Statistics for Lustre filesystems.

\begin{tabular}{r|r|rr|rr|rr|rr|rr}
\hline \multirow{2}{*}{$\boldsymbol{f}_{\boldsymbol{s}}$} & \multirow{2}{*}{ App hours } & \multicolumn{2}{|c}{ read_mb } & \multicolumn{2}{c}{ read_ops } & \multicolumn{2}{c}{ write_mb } & \multicolumn{2}{c}{ write_ops } & \multicolumn{2}{c}{ other } \\
& & $\mu$ & $c_{v}$ & $\mu$ & $c_{v}$ & $\mu$ & $c_{v}$ & $\mu$ & $c_{v}$ & $\mu$ & $c_{v}$ \\
\hline 1 & 3447 & 16585 & 4 & 150418 & 6 & 3783 & 7 & 4224 & 6 & 313150 & 6 \\
2 & 1125513 & 5427 & 13 & 28680 & 14 & 19904 & 16 & 26396 & 14 & 157789 & 12 \\
3 & 1940595 & 4452 & 26 & 14439 & 21 & 26187 & 33 & 33016 & 28 & 115807 & 18 \\
4 & 717520 & 13929 & 5 & 508683 & 11 & 22214 & 20 & 29367 & 18 & 1100889 & 10 \\
\hline
\end{tabular}

Table 2: MDS Statistics for Lustre filesystems.

\begin{tabular}{|c|c|c|c|c|c|c|c|c|c|c|c|c|c|c|c|c|}
\hline \multirow{2}{*}{$f s$} & \multicolumn{2}{|l|}{ open } & \multicolumn{2}{|c|}{ close } & \multicolumn{2}{|c|}{ mkdir } & \multicolumn{2}{|c|}{ rmdir } & \multicolumn{2}{|c|}{ getattr } & \multicolumn{2}{|c|}{ setattr } & \multicolumn{2}{|c|}{ sync } & \multicolumn{2}{|c|}{ statfs } \\
\hline & $\mu$ & $c_{v}$ & $\mu$ & $c_{v}$ & $\mu$ & $c_{v}$ & $\mu$ & $c_{v}$ & $\mu$ & $c_{v}$ & $\mu$ & $c_{v}$ & $\mu$ & $c_{v}$ & $\mu$ & $c_{v}$ \\
\hline 1 & 45391 & 9 & 45282 & 9 & 0.8 & 19 & 0.5 & 31 & 1177 & 18 & 541 & 54 & 996 & 7 & 8 & 6 \\
\hline 2 & 24314 & 17 & 22040 & 18 & 105 & 130 & 10 & 67 & 13596 & 10 & 6793 & 14 & 317 & 37 & 1.2 & 38 \\
\hline 3 & 41547 & 10 & 35389 & 12 & 40 & 22 & 16 & 29 & 13626 & 14 & 1794 & 22 & 23 & 41 & 5 & 16 \\
\hline 4 & 118166 & 6 & 76457 & 7 & 1299 & 32 & 37 & 17 & 20311 & 16 & 2287 & 14 & 32 & 31 & 3 & 29 \\
\hline
\end{tabular}

Slowdown events are usually reported to HPC support staff (ARCHER helpdesk) and historically $f_{s} 2$ has the highest number of such events, with $f_{s} 3$ seeing the second highest and $f_{s} 4$ fewer slowdowns. This does not correlate with the combined raw I/O statistics out of LASSi.

\section{LASSI}

LASSi extends the work of Diana Moise (Hoppe, Gienger, Bonisch, Shcherbakov, and Moise 2017) on the Hazel Hen system at the High Performance Computing Center Stuttgart (HLRS), which identified aggressor and victims based on "running at the same time" as an indicator. Grouping applications based on the exact command line used, the study defines slowdown as a deviation from the average run times by 1.5 times or more. This study did not use any I/O or network statistics.

Victim detection is based on observing applications that run slower than the average run time for an application group. Aggressor detection is based on applications that overlap with the victims. The aggressor and victim model based on concurrent running becomes difficult to apply when we move to a system like ARCHER, where a large number of applications are usually running. Instead, the LASSi project has defined metrics that indicate problematic behaviour. Ultimately, we have shown that there is less distinction between victims and aggressor than expected. An alternative explanation, supported by the LASSi derived data is that so-called victims are simply using the Lustre filesystem more heavily than so-called aggressors.

\subsection{Risk-Metric Based Approach}

We focus on I/O as the most likely cause of application slowdown and begin with the assumption that in isolation, slowdown only happens when an application does more I/O than expected or when an application has an unusually high resource requirement compared to normal. We expect that users will report slowdown only when their applications run at a time when the filesystem is busier than usual. 
To characterise situations that cause slowdown means considering raw $\mathrm{I} / \mathrm{O}$ rate, metadata operations and quality (size) of I/O operations. For example, Lustre filesystem usage is optimal when at least $1 \mathrm{MB}$ is read or written for each operation (read_ops or write_ops). Comparing the read_mb, write_mb with the read_ops and write_ops from Table 1, we can infer that the reads are usually sub-optimal ( $\ll 1 \mathrm{MB})$ compared to writes.

The central metadata server can sustain a certain rate of metadata operations, above which any metadata request from any application or group of applications will cause slowdown. To provide the type of analysis required, LASSi must comprehend this complex mixture of different applications with widely different $\mathrm{read} / \mathrm{write}$ patterns, the metadata operations running at the same time and how these interact and affect each other. This requirement informs the LASSi metrics definition.

\subsection{Definition of Metrics}

Metrics for quantity and quality of application I/O operations must be defined. We first define the risk for any OSS or MDS operation $x$ on a filesystem $f s$ as

$$
\operatorname{risk}_{f s}(x)=\frac{x-\alpha * \operatorname{avg}_{f s}(x)}{\alpha * a v g_{f s}(x)} .
$$

$\alpha$ is a scaling factor and is set arbitrarily to 2 for this analysis. The risk metric measures the deviation of Lustre operations from the (scaled) average on a filesystem. A higher value indicates higher risk of slowdown to a filesystem.

We introduce metrics risk ${ }_{\text {oss }}$ and risk $_{m d s}$ that accumulate risks to OSS and MDS respectively and are defined by

$$
\text { risk }_{\text {oss }}=\text { risk }_{\text {read_kb }}+\text { risk }_{\text {read_ops }}+\text { risk }_{\text {write_kb }}+\text { risk }_{\text {write_ops }}+\text { risk }_{\text {other }}
$$

and

$$
\begin{aligned}
& \text { risk }_{m d s}=\text { risk }_{\text {open }}+\text { risk } k_{\text {close }}+\text { risk }_{\text {getattr }}+\text { risk }_{\text {setattr }}+\text { risk }_{m k d i r} \\
& + \text { risk }_{\text {rmdir }}+\text { risk }_{\text {mknod }}+\text { risk }_{\text {link }}+\text { risk }_{\text {unlink }}+\text { risk }_{\text {ren }} \\
& + \text { risk }_{\text {getxattr }}+\text { risk }_{\text {setxattr }}+\text { risk }_{\text {statfs }}+\text { risk }_{\text {sync }}+r i s k_{c d r}+r i s k_{s d r} \text {. }
\end{aligned}
$$

Non-positive risk contributions are always ignored.

The above metric measures the quantity of I/O operations, but not the quality. On Lustre $1 \mathrm{MB}$ is the optimal size for read or write per operation. In order to have a measure for the quality of application reads and writes we define the metrics

$$
r e a d \_k b \_o p s=\frac{r e a d \_o p s * 1024}{r e a d \_k b}
$$

and

$$
\text { write_kb_ops }=\frac{\text { write_ops } * 1024}{\text { write_kb }} \text {. }
$$

The read or write quality is optimal when read_kb_ops $=1$ or write_kb_ops $=1$. A value of read_kb_ops $>>1$ or write_kb_ops $>>1$ denotes poor quality read and writes. In general, risk measures the quantity of I/O and ops measures the quality. 
Sivalingam, Richardson, Tate and Lafferty

\subsection{LASSi Architecture}

LASSi analytics consists of a complex workflow of data movement across different components developed in PySpark (http://spark.apache.org/docs/2.2.0/api/python/pyspark.html) - a Python API for Spark - C and Scala. I/O metrics are computed per application per hour for all three filesystems of ARCHER. They need to be computed in real-time to enable notification of users or triggering of events in the case of high risk. Figure 1 shows the architecture of LASSi and the data-flow through different components of the tool.

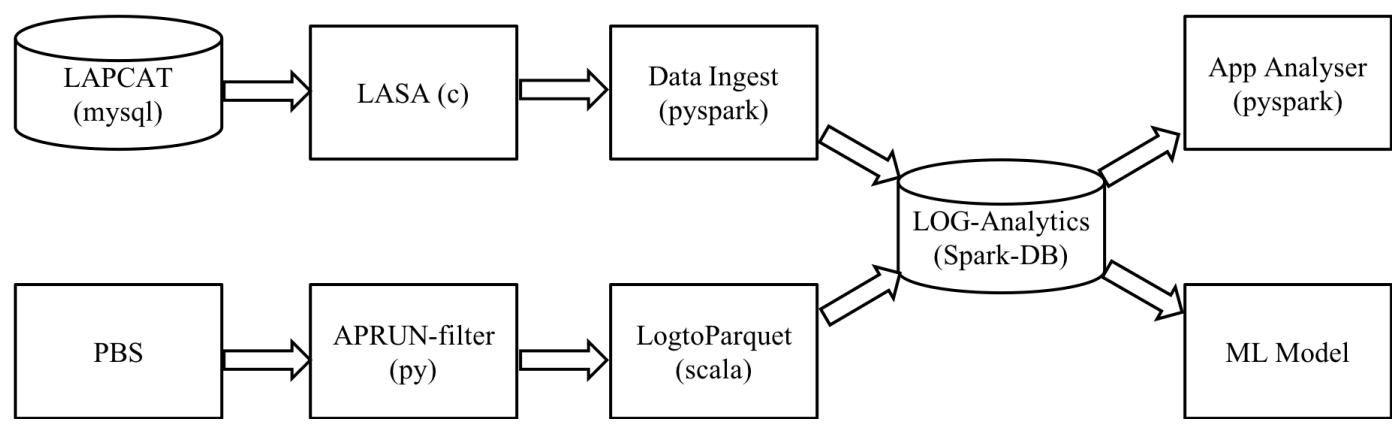

Figure 1: Architecture of LASSi showing different components and flow of data through the components.

As noted in Section 2, the I/O statistics are collected using a tool called LAPCAT at 3-minute granularity. The discrete output may result in errors in I/O statistics attribution at the start and end of application runs. On HPC machines (like ARCHER), applications usually run for many hours and sharp peaks in I/O operations do not affect the application run time compared to sustained high levels in $\mathrm{I} / \mathrm{O}$ operations. This means that the discretization errors can be easily ignored. Application details including the start time, end time and the compute node list are obtained from the job scheduler.

LASSi could analyse over 3-minute periods but this might be very expensive. For practical purposes, LASSi aggregates the data over 60 minutes for analysis. All statistics quoted below are using this hourly basis unless mentioned otherwise. LASA is a $\mathrm{C}$ application that aggregates the $\mathrm{I} / \mathrm{O}$ stats for each application over an hour and stores them in a simpler mapping from application ID to I/O statistics for every hour of its run. This data is generated in csv format.

Application ID and job ID are not informative but the exact command used to launch the application contains valuable information that can be used to group applications. This grouping was the basis of the victim-aggressor analysis for the initial work (Hoppe, Gienger, Bonisch, Shcherbakov, and Moise 2017). This quantity can be used to find average run times and then study slowdown in application performance. ARCHER uses a PBS scheduler (https://www.pbsworks.com), and APRUN-filter is a python application that filters application information including the exact command in a csv format.

Spark (Zaharia et al. 2016) is used as the data analysis and data mining engine. Spark has an in-built database that supports data import from csv files and also query using SQL. I/O statistics and job data are stored in relational tables and analysed using SQL queries. The I/O statistics generated by LASA (in csv format) are ingested by a Spark DB "Data ingest" python tool. The job data is also imported to the SparkDB using the LogtoParquet Scala script. Parquet stores the data in a vectorised format that improves the performance of Spark queries.

This data is then aggregated to obtain hourly I/O statistics for all applications running on ARCHER. The risk and ops metrics are generated for all application runs every hour by running Spark-based SQL queries. The generated risk and ops profiles are then used for analysis. LASSi also aggregates statistics for whole groups of applications based on the run command used. 
The average application run time statistic can be used to study slowdown in application runs. This metricsbased framework was developed with the intention of automating analysis on a daily basis, auto-generating plots and reports and potentially providing real-time analysis in the future. Current reporting and plots (see Section 4) are generated using python and the matplotlib library.

\section{LASSI USAGE AND ANALYSIS}

The current LASSi workflow provides daily analysis of the previous day's filesystem usage. Daily reports generated by LASSi are accessible to helpdesk and support staff. Any slowdown in application run time is usually reported to the helpdesk; the support staff can correlate reported slowdowns of applications to the generated metrics and identify the application(s) that are causing the problem. This process of triaging application issues previously consumed significant time and was often inconclusive regarding cause of slowdown. In the case of one Python application that previously caused slow filesystem response, the investigation took several days - similar conclusions can now be reached in a moment using the LASSi tool with automated daily reports.

\subsection{Daily reports}

LASSi generates daily reports showing I/O statistics and metrics of the previous day for all filesystems. The daily reports contain plots of risk_stats, $m d s \_r i s k, o s s \_r i s k$ and ops_metric. LASSi can also generate reports over a specified time period. The risk_stats plots show the MDS and OSS risk statistics for a filesytem on a certain period. Figure 2 shows a sample report showing OSS and MDS risk over 24 hours of 2017-10-10 to $f s 2$. These plots can be early indicators of potential slowdown behaviour.

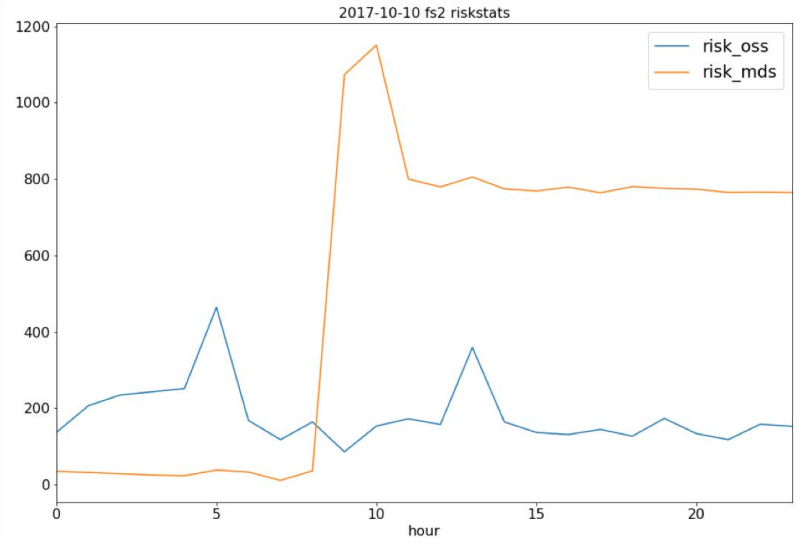

Figure 2: Sample report showing the risk (from eqns 3 and 4) to filesystem $f s 2$ over 24 hours of 2017-10-10.

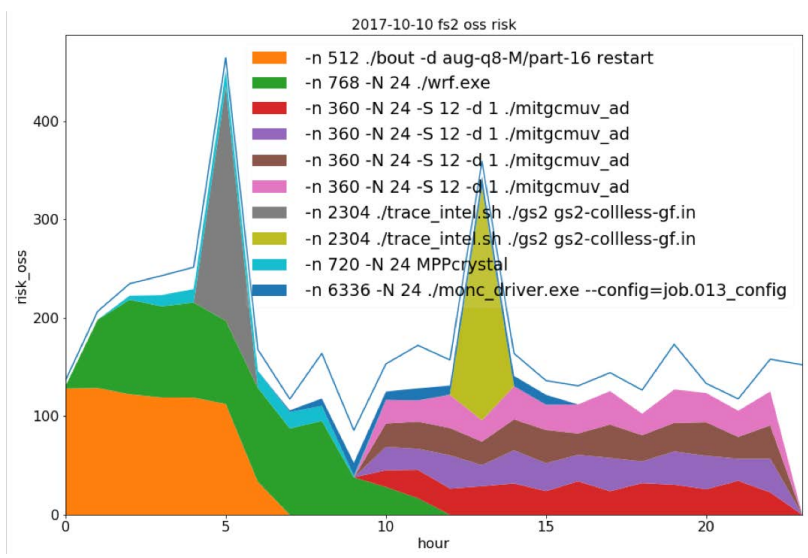

Figure 3: Sample report showing the OSS risk to filesystem $f s 2$ over 24 hours of 2017-10-10 with applications that are contributing to the risk.

The oss_risk report shows OSS risk statistics along with the applications contributing to the risk over time. Figure 3 shows a sample $o s s \_r i s k$ report for filesystem $f_{s} 2$ on 2017-10-10 and the contributing applications. Multiple different applications like bout, wrf, mitgcmuv, gs2, crystal and monc are shown to be causing risk to the filessytem at different times. We see that tracing of $g s 2$ has peaks in OSS risk, while applications like wrf and mitgcmuv have sustained risk to OSS operations. These reports helped identify multiple cases where slowdown was caused by different applications running at the same time.

The $m d s \_r i s k$ report shows MDS risk statistics along with the applications contributing to the risk over time. Figure 4 shows a sample $m d s \_r i s k$ report for filesystem $f s 2$ on 2017-10-10 and the contributing applications. 
This is different from the risk_oss plot as we see tasks in a taskfarm contributing to the risk_mds. Each task contributes to the overall high risk and these are very hard to study and analyse in isolation. Note that these are not always submitted from a single job or job array. We have already identified a pattern of 'task farm'-like applications with similar I/O requirements scheduled at the same time causing considerable risk and slowdown.

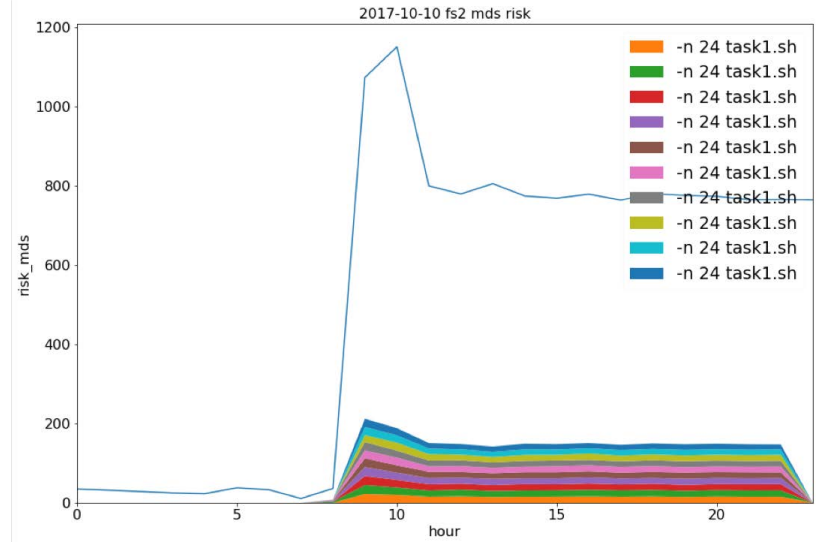

Figure 4: Sample report showing the MDS risk to filesystem $f s 2$ over 24 hours of 2017-10-10 with applications that are contributing to the risk.

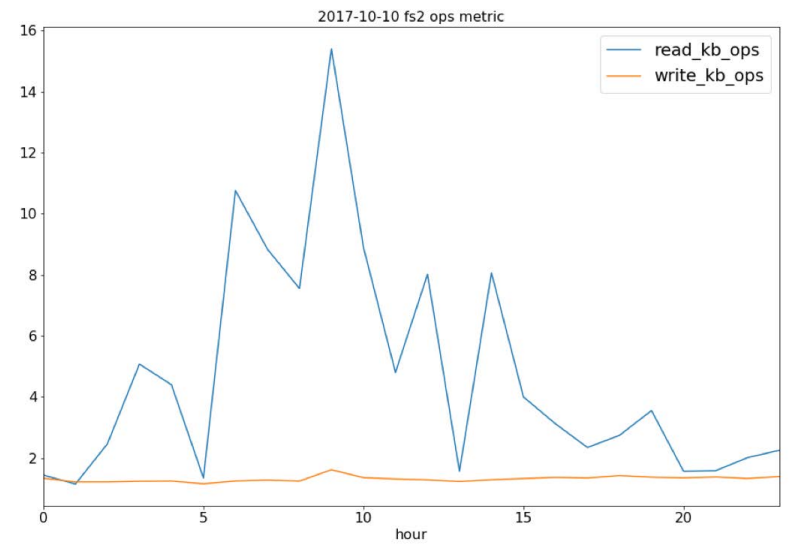

Figure 5: Sample report showing the read and write quality (from eqns 5 and 6 ) to filesystem $f s 2$ over 24 hours of 2017-10-10.

The ops_metric report shows read and write ops statistics for a filesystem over time. Figure 5 shows the read_kb_ops and write_kb_ops metrics for $f s 2$ on 2017-10-10. We observe that the writes are near optimal whereas the reads are sub-optimal at different time periods. This is a recurring feature in our analysis as application read quality is usually suboptimal compared to the quality of writes.

Reports allow HPC support staff to identify and triage the exact time of risk and the applications that cause risk of slowdown. In the case of high OSS risk, attention should be given to the quality of reads and writes to ensure that Lustre is optimally used. We observed one tracing application writing a few bytes every second to Lustre, which is clearly suboptimal and the problem was resolved by buffering into scratch space. In case of high MDS risk, the application should be carefully studied for high metadata operations that contribute to the risk. One incorrectly configured application was creating millions of directories per second and this was easily identified using the metrics. This information is usually passed to the application owner or deep technical support available as part of the ARCHER service who can engage directly with the user.

In addition to daily monitoring, studying the metrics of the filesystem helps us understand standard usage of filesystems, define application classes from an I/O perspective and identify general issues in I/O usage on the system.

\subsection{Application slowdown analysis}

The LASSi risk and ops metrics we have defined should capture the application slowdown. Through these metrics and the associated reports, LASSi can identify application slowdown and assist root cause diagnosis. All metrics are designed such that higher values are not optimal. Optimal values for risk and ops metrics are 0 and 1 respectively. The main contribution factor for slowdown of an application is the I/O load (characterised by the metrics) of the filesystem and the I/O profile of that application at any time. Applications performing no reads and writes will not be impacted by the I/O load in a filesystem. 


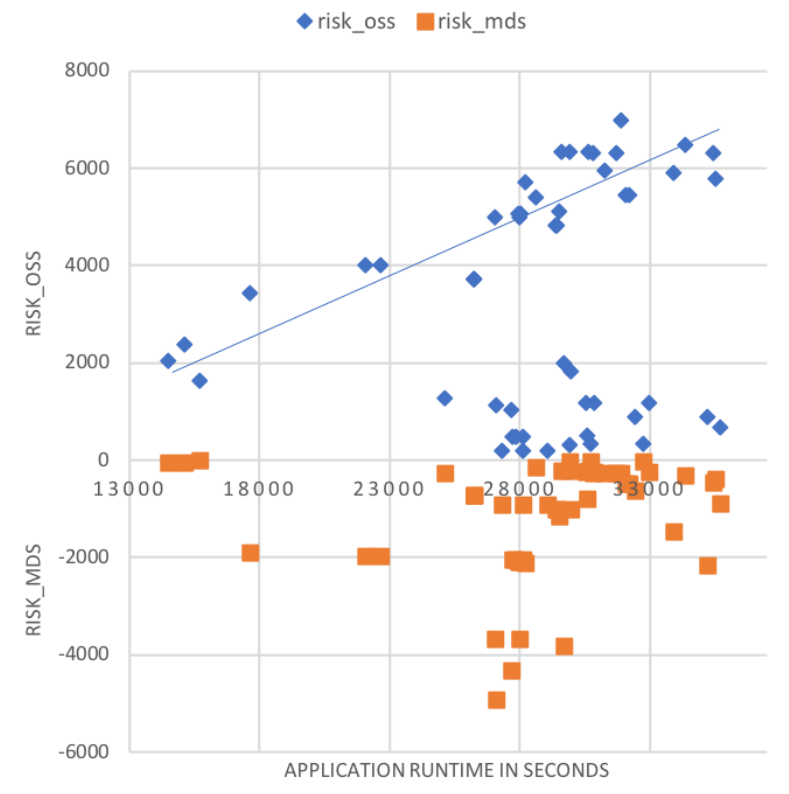

Table 3: OSS and MDS risk to filesystem during job runtime.

\begin{tabular}{||ccc||}
\hline Job & risk_oss & risk_mds \\
\hline \hline job1 & 502 & 77 \\
job2 & 502 & 77 \\
job3 & 502 & 77 \\
job4 & 502 & 77 \\
job5 & 118 & 544 \\
job6 & 282 & 824 \\
job7 & 164 & 280 \\
job8 & 164 & 280 \\
\hline
\end{tabular}

Figure 6: Scatter plot of application run time vs risk of the filesystem for a set of weather/climate jobs.

LASSi was partly designed to assist in understanding situations where users report performance variation (slowdown) of similar runs. There have been many such incidents reported in ARCHER and we have successfully mapped application slowdown to high risks in filesystems at the time in question. The application(s) causing high risk are then studied in detail to improve the I/O usage. For reported performance variation, we depend on the application owner to clearly label similar job runs and identify slow run times.

For example, a user complained about performance variation over 2 days for a Computational Fluid Dynamics $(C F D)$ application. Table 3 shows the sum of risks to the file system during the job run time. Jobs 1 to 4 ran normally whereas jobs 5 to 8 ran slowly. The slowdown can be directly mapped to the high metadata risk in the filesystem during the run times. The high risk to OSS does not affect these $C F D$ applications. Using LASSi we can also study the coarse application profile and this $C F D$ application was found to be doing thousands of meta-data operations (open and close) within each second. The high MDS risk to filesystem was caused by taskfarm applications running in parallel. Thus we can map the slowdown to the I/O profile of the application and the I/O load of the filesystem.

Grouping application runs is very difficult and usually requires the input of the application owner to label the runs that are expected to have similar run time. LASSi metrics can be correlated with the run time of application runs, by grouping based on the exact command used to launch the application. The launch command usually includes node count, exact node configurations like threads per core, application executable and application arguments.

Figure 6 shows the scatter plot of application run time vs the encountered risk_oss (positive axis) and risk_$m d s$ (negative axis) in the filesystem for a set of climate and weather jobs. Here risk metrics are summed over the run time of each application run. The superimposed line in the plot shows a possible linear relationship between risk_oss and run times. This application group used here has an average runtime of 13500 seconds and reads $106 \mathrm{MB}$, writes $14.2 \mathrm{~GB}$ and performs $33 \mathrm{~K}$ metadata operations per hour. The average read and write quality are 1.2 and 2.1 and are close to optimal. All these application runs have zero risk with I/O statistics well below the filesystem average. 
From the plot, we can see higher OSS and MDS risk on the filesystem when jobs with run time more than 13500 s were running, with a cluster showing a possible linear relationship for risk_oss and application run time. The high OSS risk was found to be caused by a python application that was reading and writing a few bytes per second at that time. There is also a cluster of jobs with lesser OSS risk having a run time of more than 23500s which cannot be explained from the risk metrics alone. A complete analysis is not possible without understanding the application's science, I/O profile and network bandwidth of each job run. This slowdown analysis did not require the input of the application owner, unlike the previous analysis.

Although LASSi only considers I/O statistics, it has been successful in modeling and resolving slowdown incidents reported by application users for over 6 months. In all cases applications causing slowdown have been identified using risk and ops metrics and appropriate remedial action had been taken. This approach is more generally applicable to any environment with a shared filesystem as long as the relevant data can be collected.

\section{RELATED WORK}

UMAMI (Lockwood et al. 2017) uses an approach of analysing I/O statistics using meaningful metrics in a similar fashion to LASSi. They stress a need for a holistic I/O analysis as their metrics do not capture enough details to indicate performance loss. MELT (Brim and Lothian 2015), a unified Lustre performance monitoring and analysis infrastructure tool, helps administrators analyse reported application slowdowns by providing command line utilities to view I/O statistics of clients, servers and jobs. Using MELT requires expertise and does not provide an automatic root cause analysis solution for performance problems. ldiskfs (Laifer 2015) is a tool for generating Lustre I/O stats for jobs. The script runs hourly and collects and summarises the jobs I/O stats and then mails the user. Lustre Monitoring Tool (LMT) (Lustre 2018) is an open-source tool for capture and display of Lustre file system activity. I/O statistics are stored in a MySQL database with command line utilities for live monitoring. LMT does not map I/O statistics to jobs. Kunkel et al. (Kunkel, Betke, Bryson, Carns, Francis, Frings, Laifer, and Méndez 2018) review existing tools for analysing I/O performance of parallel system and online monitoring tools developed at DKRZ and LLView by LLNL. They reveal how these tools can be used to study I/O issues. Mendez et al. (Mendez et al. 2017) evaluated I/O performance of applications as a function of I/O characteristics and performance capacity of the I/O system by defining a metric called I/O severity. This metric identifies the factors limiting the I/O performance of a kernel or application but does not study the effects of multiple applications interacting with the I/O system. Researchers at NERSC (Uselton and Wright 2013) introduced a new metric named File System Utilisation (FSU) based on series of calibration experiments using IOR, to study I/O workload on the file system. Many monitoring tools (Uselton and Wright 2013), (Uselton 2009), (Shipman, Dillow, Oral, Wang, Fuller, Hill, and Zhang 2010), (Uselton, Antypas, Ushizima, and Sukharev 2010), and (Miller, Hill, Dillow, Gunasekaran, Shipman, and Maxwell 2010) for raw I/O statistics of filesystems and jobs have been used to study and improve I/O performance of applications. The tools described above provide raw I/O statistics of filesystem or applications. LASSi moves beyond this by delivering a framework where it is easy to identify applications with unusual I/O behaviour, and by targeting application interactions with the filesystem. LASSi is an non-invasive approach that does not perturb the filesystem. Additionally, LASSi provides holistic I/O analysis by monitoring filesystem I/O, generating coarse profiles of filesystems and application runs in time and automating analysis of application slowdown using metrics. LASSi can also be used to study I/O patterns of application groups which is important for those that manage filesystems.

\section{CONCLUSION}

LASSi is a tool primarily designed to help HPC support staff triage and resolve issues of application slowdown due to contention in a shared filesystem. LASSi uses a metrics-based analysis in which risk and ops metrics correlate to the quantity and quality of an application's I/O. The tool's workflow is automated to 
produce near real-time analysis of filesystem health and application I/O profiles. Using the metrics and analysis, LASSi is being used to study the I/O profile of applications, understand common I/O usage of application groups, locate the reasons for slowdown of similar jobs and to study filesystem usage in general. For example we have identified a particular class of jobs (task farms) that can generate excessive I/O load even though individual applications are not a concern. This information can be used not only to optimise applications and avoid slowdown but also in the planning and configuration of the HPC filesystem for different projects. We have shown that the application-centric non-invasive approach based on metrics that is used by LASSi is valuable in understanding application I/O behaviour in a shared filesystem.

\section{FUTURE WORK}

ARCHER support staff continue to monitor the LASSi metrics against reported application slowdown and contact application owners of rogue applications to better understand and optimise their I/O. Using these reported incidents, LASSi metrics are continuously improved and tuned or new metrics added. Currently our analysis uses a coarse time resolution of 1 hour, we plan to move to a 6 minute window with hourly analysis of filesystem health. The ideas from this work can also be ready applied for network statistics and this will be explored in the future.

\section{ACKNOWLEDGMENT}

This work was undertaken by the Cray Centre of Excellence for ARCHER funded by EPSRC. We would like to acknowledge EPSRC, Cray, ARCHER User Support and User Community for their support.

\section{REFERENCES}

Braam, P. J. et al. 2003. "The Lustre storage architecture”. White Paper, Cluster File Systems, Inc., Oct vol. 23.

Brim, M. J., and J. K. Lothian. 2015. "Monitoring Extreme-scale Lustre Toolkit". CoRR vol. $\mathrm{abs} / 1504.06836$.

Cray 2018. "Cray XC Series Supercomputers". https://www.cray.com/products/computing/xc-series.

EPCC 2018. "ARCHER Virtual Tutorials and Webinars". http://www.archer.ac.uk/training/virtual/. Accessed Dec. 12, 2018.

Henty, David 2018. "Efficient Parallel IO on ARCHER @ EPCC at Cambridge”. https://events.prace-ri.eu/ event/696/. Accessed Dec. 12, 2018.

Hoppe, D., M. Gienger, T. Bonisch, O. Shcherbakov, and D. Moise. 2017. "Towards Seamless Integration of Data Analytics into Existing HPC Infrastructures". Proc. Cray Users Group.

Karo, M., R. Lagerstrom, M. Kohnke, and C. Albing. 2006. "The application level placement scheduler". Cray User Group, pp. 1-7.

Kunkel, J. M., E. Betke, M. Bryson, P. H. Carns, R. Francis, W. Frings, R. Laifer, and S. Méndez. 2018. “Tools for Analyzing Parallel I/O”. CoRR vol. abs/1807.04985.

Laifer, Roland 2015. "Lustre tools for ldiskfs investigation and lightweight I/O statistics". http://www.scc. kit.edu/scc/docs/Lustre/kit_lad15_20150922.pdf. Accessed Dec. 12, 2018.

Lockwood, G. K. et al. 2017. "UMAMI: A Recipe for Generating Meaningful Metrics Through Holistic I/O Performance Analysis". In Proceedings of the 2nd Joint International Workshop on Parallel Data Storage \& Data Intensive Scalable Computing Systems, PDSW-DISCS '17, pp. 55-60. New York, NY, USA, ACM. 
Lustre 2018. "Lustre Monitoring and Statistics Guide". Accessed Dec. 12, 2018.

Mendez, S. et al. 2017. "Analyzing the Parallel I/O Severity of MPI Applications". In Proceedings of the 17th IEEE/ACM International Symposium on Cluster, Cloud and Grid Computing, CCGrid '17, pp. 953-962. Piscataway, NJ, USA, IEEE Press.

Miller, R., J. Hill, D. A. Dillow, R. Gunasekaran, G. M. Shipman, and D. Maxwell. 2010. "Monitoring tools for large scale systems". In Proceedings of Cray User Group Conference (CUG 2010).

NASA GOV 2018. "Lustre Best Practices". https://www.nas.nasa.gov/hecc/support/kb/ lustre-best-practices_226.html. Accessed Dec. 12, 2018.

National Computational Infrastructure, Australia 2018. "Lustre Best Practices - NCI Help". https://opus.nci. org.au/display/Help/LustreBestPractices.

NICS 2018. "I/O and Lustre Usage". https://www.nics.tennessee.edu/computing-resources/file-systems/ io-lustre-tips\#io-best-practices. Accessed Dec. 12, 2018.

Shipman, G., D. Dillow, S. Oral, F. Wang, D. Fuller, J. Hill, and Z. Zhang. 2010. "Lessons learned in deploying the world's largest scale Lustre file system". In The 52nd Cray user group conference.

Turner, Andy and others 2017. "Parallel I/O Performance". https://www.archer.ac.uk/training/virtual/ 2017-02-08-Parallel-IO/2017_02_ParallelIO_ARCHERWebinar.pdf. Accessed Dec. 12, 2018.

Uselton, A. 2009. "Deploying server-side file system monitoring at NERSC". Technical report, Lawrence Berkeley National Lab.(LBNL), Berkeley, CA (United States).

Uselton, A., K. Antypas, D. Ushizima, and J. Sukharev. 2010. "File system monitoring as a window into user I/O requirements". In Proceedings of the 2010 Cray User Group Meeting, Edinburgh, Scotland. Citeseer.

Uselton, A., and N. Wright. 2013. "A file system utilization metric for I/O characterization". In Proc. of the Cray User Group conference.

Zaharia, M. et al. 2016, October. "Apache Spark: A Unified Engine for Big Data Processing”. Commun. $A C M$ vol. 59 (11), pp. 56-65.

\section{AUTHOR BIOGRAPHIES}

KARTHEE SIVALINGAM is a Research Engineer at the Cray EMEA Research Lab. He is part of the Cray Center of Excellence for ARCHER that engages with users to allow them to maximise their use of Cray technologies. He has particlar interest in I/O, Workflows, Optimisation, overlap of HPC with Big data and AI ksivalinga@cray.com.

HARVEY RICHARDSON is a Senior Research Engineer at the Cray EMEA Research Lab. He works on EU-funded research projects and the Cray Centre of Excellence for ARCHER. He has particular interests in computer architecture and performance, programming models and language standards.

ADRIAN TATE is Principal Researh Engineer and Director of the Cray EMEA Research Lab. He is the technical coordinator of the EU Maestro project and is involved in several other EU-funded projects, mostly related to efficient usage of memory hierarchy adrian@cray.com.

MARTIN LAFFERTY is a Senior Systems Engineer at the Cray UK Ltd. His work is currently focused around the ARCHER supercomputer based at Edinburgh University with occasional involvement in other global projects. His main interests are computer architecture, I/O performance, system optimisation, monitoring tools, archival and complex systems firefighting rml@cray.com. 